\title{
Antimicrobial Activity of Lactobacilli against Staphylococcus aureus MRSA Strains Isolated from Mastitis in Dairy Animals
}

\author{
Jyotika D. Sangle, Prashant P. Mhase*, Mrunalini M. Pawade and Prerana R. Shelke \\ Department of Veterinary Microbiology, KNPCV, Shirwal, MAFSU, Nagpur(MS), India \\ *Corresponding author
}

\section{A B S T R A C T}

\begin{tabular}{|l|}
\hline K e y w or d s \\
$\begin{array}{l}\text { Mastitis, MRSA, } \\
\text { Lactobacilli, } \\
\text { Antimicrobial activity }\end{array}$ \\
\hline Article Info \\
\hline $\begin{array}{l}\text { Accepted: } \\
\text { 26 October } 2018 \\
\text { Available Online: } \\
10 \text { November } 2018\end{array}$ \\
\hline
\end{tabular}

Methicillin resistant Staphylococcus aureus (MRSA) strains being a major pathogen associated with mastitis of dairy animals, the study was proposed for investigating antimicrobial activity of lactobacilli of dairy animals having activity against the MRSA isolates of subclinical mastitis. Twenty two Lactobacilli were isolated from milk of healthy animal and screened for inherent antimicrobial activity with agar spot technique against indicator strain L. acidophilus, out of which eleven isolates exhibited strong inhibitory activity. The cell free filtrates of these isolates were then tested in vitro against MRSA by employing agar well diffusion and agar disc diffusion tests. Zone size indicative antimicrobial activity against MRSA possessing a very strong $(15-18 \mathrm{~mm})$ to moderately strong (10-14 mm) efficacy was observed in L. plantarum strains belonging to buffaloes (B5 and B8) and goats (G2 and G6), followed by sheep (S3). Moderate inhibitory activity (6-9 $\mathrm{mm})$ was also observed for L. paraplantarum strain from cow (C2).

\section{Introduction}

Bovine mastitis is an economically most important disease of dairy industry which is thriving on a very marginal profit in India. Especially subclinical form of disease in the dairy animals is very widely prevalent and is responsible for heavy losses due to reduced milk production and also has public health significance (Esslemont et al., 2000). The major pathogen associated with bovine mastitis is S. aureus. Non judicious use of the over the counter antimicrobial in treatment of subclinical mastitis (SCM) has led to a risk of emergence of antimicrobial resistant (AMR) phenotypes. Emergence of increasing AMR in coagulase positive Staphylococci (COPS) associated with mastitic cows against several antimicrobial agents has been reported earlier (Asfour and Darwish, 2011; Chandrasekaran et al., 2014) and emerging multiple AMR especially for methicillin and vancomycin group is known worldwide. Lactic acid bacteria (LAB) having property to produce lactic acid from milk sugars, constitute the predominant micro flora of healthy animal milk. LAB are well known for their use as probiotics and prebiotics in human and veterinary medicine and proved to be producing antibacterial substances like bacteriocin that replace them inhibiting the growth of pathogenic bacteria (Eid et al., 2016). CSF of LAB contains highly specific antibacterial proteins presenting a broad range 
of antimicrobial activity against pathogens resulting from production of organic acids, hydrogen peroxide, diacetyl, inhibitory enzymes as well as the bacteriocin (Mohankumar and Murugalatha, 2011).

\section{Materials and Methods}

\section{Staphylococcus aureus from subclinical mastitis}

Total 100 dairy animals comprised of cow $(n=40)$ and buffaloes $(n=60)$ belonging to geographical area of Pune and Satara districts of Western Maharashtra, India, presented for SCM were screened using California mastitis test (CMT). Each of CMT positive (60) milk samples was inoculated in BHI broth and incubated at $37^{\circ} \mathrm{C}$ for 24 hours. The cultures were streaked on nutrient agar and incubated at $37^{\circ} \mathrm{C}$ for 24 hours. Smears of identical colonies were stained and only gram positive cocci found in clusters were further processed for selective growth of $S$. aureus strains on mannitol salt agar (MSA) and incubated at $37^{\circ} \mathrm{C}$ overnight. Characteristic yellowish colonies on MSA were considered as presumptive $S$. aureus and maintained in pure culture. Phenotypic typing of $S$. aureus was performed by staining, cultural characteristics and biochemical tests (Quinn et al., 2002).

\section{Antibiogram profile of Staphylococcus} aureus

Antibiotic sensitivity profiles of $S$. aureus isolates as well as other organisms were studied against nine different antibiotics. Antibiotics were randomly selected preferably considering their use in field for treatment of mastitis by clinicians in our study area and performed by the disc diffusion technique Bauer et al., (1966) with minor modifications (CLSI, 2014). The commercially available antibiotic discs used were methicillin $(10 \mathrm{mcg})$, vancomycin $(10 \mathrm{mcg})$, ciprofloxacin $(5 \mathrm{mcg})$, enrofloxacin $(10 \mathrm{mcg})$, gentamycin $(10 \mathrm{mcg})$, chloramphenicol (30 mcg), amoxicillin/clavonic acid (20/10 mcg), ceftriaxone/tazobactum (30/10 mcg) and penicillin G (10unit) were procured from HiMedia Pvt Ltd., India.

\section{LAB from raw milk samples}

Raw unpasteurized milk samples ( $\mathrm{n}=10$ each) from apparently healthy CMT negative cow, buffalo, goat and sheep were collected from the animals belonging to study area, aseptically. Methodology for harvesting of LAB was performed as described by Mohankumar and Murugalatha (2011). Milk samples were serially diluted in peptone medium and incubated at $23^{\circ} \mathrm{C}$ for $30 \mathrm{~min}$ before plating and diluted samples were plated onto De Mann Rogosa Sharpe (MRS) medium plates which were incubated at $37^{\circ} \mathrm{C}$ for $48-72$ hours, for isolation of LAB. Characteristic small $(2-3 \mathrm{~mm})$ colonies having pure white colour and with entire margin were picked up from each plate and transferred to MRS broth. Purity of the isolates was checked by streaking several times and sub culturing on fresh MRS agar, as well as MRS broth. The isolates were designated as C (cow), B (buffalo), G (goat) and $S$ (Sheep). Identification of LAB was attempted upto species level according to their morphological, cultural, physiological and biochemical characteristics like gram reaction, production of catalase, carbohydrate fermentation, growth at $15^{\circ} \mathrm{C}$ and $45^{\circ} \mathrm{C}$ (Bergey's Manual of Systemic Bacteriology, 1986)

\section{MALDI-TOF MS analysis of LAB}

The bacterial DNA of LAB was extracted according to the procedure of Behiry et al., (2014). $1 \mu 1$ supernatant was taken in sterile pipette and directly applied onto each spot of ground steel MSP target plate of MALDI-TOF and were air dried. Matrix $(1 \mu 1)$ was overlaid 
on these spots, air-dried and plates were introduced into the mass spectrometer for analysis. Results were displayed as a series of lines (spectrum) corresponding to different fragment of protein. Measurements were performed on a Microflex LT MALDITOF/TOF Mass Spectrometer equipped with a smart beam laser. Spectra were recorded in the linear, positive mode at a frequency of $200 \mathrm{~Hz}$ within a mass range from 2,000 to $20,000 \mathrm{Da}$. Once a spectrum were generated and captured by the software, the whole identification process was performed automatically, without any user intervention. Each peak list generated was matched directly against reference libraries $(4,623$ species) using the integrated pattern-matching algorithm of the Biotype OC 3.1.66 software. The peak intensity $\geq 1.5$ was considered at level of genus and probable species

\section{Detection of antimicrobial activity of $\mathrm{LAB}$ by Agar spot test}

Pure colonies of LAB were subcultured in 5 $\mathrm{ml}$ of MRS broth at $30^{\circ} \mathrm{C}$ for 16 hours. Aliquots $(2 \mu 1)$ of the culture were spotted onto agar plates containing $10 \mathrm{ml}$ of MRS medium. After 18 hours at $30^{\circ} \mathrm{C}$, the plates were overlaid with the $5 \mathrm{ml}$ of the appropriate soft agar $(1 \%)$ inoculated with the cell suspension of the indicator strain of L. acidophilus ATCC $4356\left(10^{5} \mathrm{CFU} / \mathrm{ml}\right)$. The plates were incubated at $37^{\circ} \mathrm{C}$ for $24-48$ hours, depending on the growth of the indicator strain and the diameters of zones of inhibition were measured. Zone size wider than $2 \mathrm{~mm}$ was scored positive.

\section{Antimicrobial efficacy of LAB against MRSA}

Each LAB strain showing efficacy in Agar spot assay were selected as potential candidates and their CFS were prepared for further evaluation as per Norroozi and Mirzaii
(2004). Isolates were cultivated in MRS broth for $24-48$ hours at $37^{\circ} \mathrm{C}$. CFS was obtained by centrifuging the culture at $10000 \mathrm{rpm}$ for 10 min followed by filtration of the supernatant through a $0.2 \mu \mathrm{m}$ pore size filter.

\section{Agar well diffusion test}

Efficacy of LAB was investigated by agar well diffusion method as per Mohankumar and Murugalatha (2011). An overnight incubated fresh culture of MRSA was prepared. These bacteria $\left(10 \mathrm{cfu} / \mathrm{ml}^{-1}\right)$ were inoculated by streaking the swab over the entire MHA surface. Around $6 \mathrm{~mm}$ diameter wells were punched on pre-inoculated agar plates and $100 \mu 1$ of each CFS of LAB was placed into each well after neutralization with $\mathrm{NaOH}$. The plates were incubated for 24 hours at $37^{\circ} \mathrm{C}$ and inhibition of growth was examined by clear zone surrounding each well.

\section{Agar disc diffusion test}

Each of the bacterial isolates were subjected to in-vitro antibacterial sensitivity testing by disc diffusion technique with different concentrations like $10 \mu 1,20 \mu$, and $30 \mu \mathrm{l}$ of supernatant as per the method of Bauer et al., (1996). In this method sterile paper disc $(6 \mathrm{~mm})$ were placed over MHA agar plates seeded with MRSA, and $30 \mu \mathrm{l}$ of cell free supernatant was added to the sterile paper disc. Plates were incubated at $37^{\circ} \mathrm{C}$ for $48 \mathrm{hrs}$. After incubation antimicrobial activity was measured in diameter $(\mathrm{mm})$ around the paper disc as per the method of Tajehmiri et al., (2014).

\section{Results and Discussion}

Detection of bovine subclinical mastitis with cultural method

Total 60 CMT positive (> 1 CMT) milk samples were collected aseptically that were 
processed for bacterial culture as described by the National Mastitis Council (1999). The results of bacterial isolation, in the milk samples from Pune region 09/20 (45.00\%) cow and 14/25 (56.00\%) buffaloes were found positive. While from Satara region 07/20 (35.00\%) cow and 27/35 (77.14\%) buffaloes yielded positive results. Overall $16 / 40(4000 \%)$ cow and 41/60 (68.33\%) buffaloes tested culturally positive. Thus, out of total 100 animals tested $60(60.00 \%)$ were CMT positive and $57(57.00 \%)$ were culturally found positive for mastitis thus, CMT test remained 95.00 percent in agreement with cultural method. Similar inferences were drawn by Sharma et al., (2007) and Saidi et al., (2013) in their studies on subclinical mastitis in bovines.

\section{Phenotypic characterization of Staphylococcus aureus from bovine subclinical mastitis}

Amongst all culturally positive samples 35/57 $(61.40 \%)$ isolates were phenotypically identified as Staphylococcus spp., amongst which 29/57 (50.87\%) were typed as $S$. aureus on MSA and remaining 06/57 (10.53\%) were minor gram positive cocci. Our findings of staphylococci, especially S.aureus as major pathogen in SCM were in close accordance with Hamed et al., (2014).

\section{Antibiogram profile of organisms associated with SCM}

In addition to 35 staphylococci the other 22/57 isolates included other $09(15.79 \%)$ non coccoid gram positive and $13(22.81 \%)$ gram negative bacteria. All these isolates were processed for their antibiogram profiling and the overall results of antimicrobial sensitivity profile as depicted indicated gentamicin as most effective drug against 25/57 (43.85\%) isolates, followed by ciprofloxacin against 22/57 (38.59\%), ceftriaxone + tazobactum against 19/57 (33.33\%), chloramphenicol against 18/57 (31.57\%), enrofloxacin against 16/57 (28.07), methicillin against 11/57 (19.29\%), amoxicillin + clavulanicacid against 09/57 (15.78\%), vancomycin against 07/57 $(12.28 \%)$ and penicillin against none (zero \%) of these isolates. Profile exhibited by $S$. aureus indicated highest resistance towards methicillin in $26 / 29(89.60 \%)$ its isolates followed by enrofloxacin in 20/29 (68.96\%), vancomycin and gentamicin in $18 / 29$ $(62.06 \%)$ each, chloramphenicol in $17 / 29$ $(58.60 \%)$, ceftriaxone + tazobactum and amoxicillin + clavulanic acid in $15 / 29$ each $(51.72 \%)$ and ciprofloxacin resistance in $08 / 29$ $(27.58 \%)$ isolates, respectively. Total 26/29 $(75.80 \%)$.

In close agreement with Gentilini et al., (2000) and Jian ping et al., (2009), very high percentage of $S$. aureus were identified as MRSA which also exhibited a very high prevalence of multiple drug resistant (MDR) strains. In all 46.00 percent $S$. aureus exhibited multiple antibiotics resistance for two to five different antibiotics used.

\section{Phenotypic characterization of LAB isolated from milk samples}

Total 40 raw milk samples were processed for isolation of LAB, 22 had resulted positive. From cow milk samples, 04/10 (40.00\%) LAB were isolated while from buffaloes milk 09/10 $(90.00 \%)$ LAB were grown. The milk samples of sheep could generate 03/10 (30.00\%) LAB isolates while goats generated $06 / 10(60.00 \%)$. This outcome was in concurrence with results published by Nair and Surendaran (2005). All LAB $(n=22)$ were attempted for identification by growth and morphological characteristics and biochemical tests as per Bergey's Manual of Determinative bacteriology (Table 1). Results of biochemical tests indicated these isolates as citrate positive and negative in MR$\mathrm{VP}$, indol, catalase and citrate. 
Table.1 Results of sugar fermentation tests performed for identification of lactobacilli isolated from raw milk

\begin{tabular}{|c|c|c|c|c|c|c|c|c|c|c|c|c|c|c|c|c|c|c|c|}
\hline $\begin{array}{l}\text { Sr } \\
\text { no }\end{array}$ & 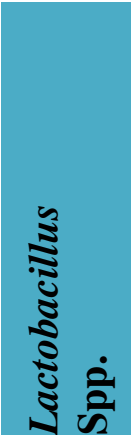 & 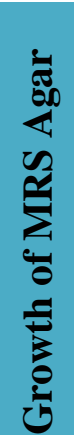 & 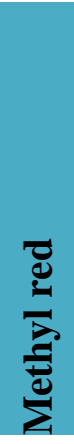 & 5 & 울 & $\frac{\tilde{\sigma}}{\frac{\pi}{\sigma}}$ & $\frac{0}{0}$ & نِّ & $\frac{\mathscr{0}}{\stackrel{0}{0}}$ & 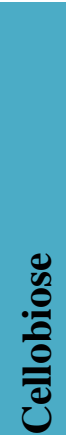 & 总 & 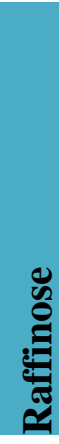 & 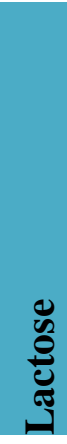 & 总 & $\frac{0}{\frac{0}{0}}$ & $\begin{array}{l}\overline{0} \\
: \frac{0}{0} \\
\text { क } \\
\text { क }\end{array}$ & $\frac{0}{\frac{\delta}{0}}$ & 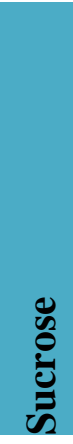 & $\begin{array}{l}\text { Species } \\
\text { Identified }\end{array}$ \\
\hline 1 & C1 & + & - & - & - & - & - & + & - & + & + & + & - & + & + & - & + & + & L. plantarum \\
\hline 2 & $\mathrm{C2}$ & + & - & - & - & - & - & + & - & + & + & + & - & + & + & - & + & + & L. plantarum \\
\hline 3 & C8 & + & - & - & - & - & - & + & - & + & + & + & - & + & + & - & + & + & L. plantarum \\
\hline 4 & C10 & + & - & - & - & - & - & + & - & + & + & + & - & + & + & - & + & + & L.plantarum \\
\hline 5 & B1 & + & - & - & - & - & - & + & + & + & + & - & + & + & - & - & + & + & L.plantarum \\
\hline 6 & B2 & + & - & - & - & - & - & + & - & + & + & + & - & + & + & - & + & + & L.plantarum \\
\hline 7 & B3 & + & - & - & - & - & - & - & + & - & - & + & d & - & + & d & + & - & Pediococcus Spp \\
\hline 8 & B4 & + & - & - & - & - & - & + & + & + & + & - & + & + & - & - & + & + & L.plantarum \\
\hline 9 & B5 & + & - & - & - & - & - & + & + & - & - & + & d & - & + & d & + & - & Pediococcus Spp \\
\hline 10 & B6 & + & - & - & - & - & - & + & d & + & + & + & - & + & + & - & + & d & $\begin{array}{l}\text { Other Lactobacillus } \\
\text { spp }\end{array}$ \\
\hline 11 & B8 & + & - & - & - & - & - & - & + & - & - & + & d & - & + & d & + & - & Pediococcus Spp \\
\hline 12 & B9 & + & & & & & & & & & & & & & & & & & $\begin{array}{l}\text { Other Lactobacillus } \\
\text { spp }\end{array}$ \\
\hline 13 & B10 & + & - & - & - & - & - & + & d & + & + & + & - & + & + & - & + & d & $\begin{array}{l}\text { Other Lactobacillus } \\
\text { spp. }\end{array}$ \\
\hline
\end{tabular}




\begin{tabular}{|c|c|c|c|c|c|c|c|c|c|c|c|c|c|c|c|c|c|c|c|}
\hline 14 & G1 & + & - & - & - & - & - & + & - & + & + & + & - & + & + & - & + & + & L.plantarum \\
\hline 15 & G2 & + & - & - & - & - & - & + & - & + & + & + & - & + & + & - & + & + & L.plantarum \\
\hline 16 & G3 & + & - & - & - & - & - & + & - & + & + & + & - & + & + & - & + & + & L.plantarum \\
\hline 17 & G5 & + & - & - & - & - & - & + & - & + & + & + & - & + & + & - & + & + & L.plantarum \\
\hline 18 & G6 & + & - & - & - & - & - & + & d & + & + & + & - & + & + & - & + & d & $\begin{array}{l}\text { Other Lactobacillus } \\
\text { spp }\end{array}$ \\
\hline 19 & S1 & + & - & - & - & - & - & - & + & - & - & + & d & - & + & d & + & - & Pediococcus Spp \\
\hline 20 & S3 & + & - & - & - & - & - & + & d & + & + & + & - & + & + & - & + & d & $\begin{array}{l}\text { Other Lactobacillus } \\
\text { spp }\end{array}$ \\
\hline 21 & S9 & + & - & - & - & - & - & + & - & + & + & + & - & + & + & - & + & + & L.plantarum \\
\hline 22 & S10 & + & - & - & - & - & - & + & - & + & + & + & - & + & + & - & + & + & L.plantarum \\
\hline 13 & B10 & + & - & - & - & - & - & + & d & + & + & + & - & + & + & - & + & d & $\begin{array}{l}\text { Other Lactobacillus } \\
\text { spp. }\end{array}$ \\
\hline 14 & G1 & + & - & - & - & - & - & + & - & + & + & + & - & + & + & - & + & + & L.plantarum \\
\hline 15 & G2 & + & - & - & - & - & - & + & - & + & + & + & - & + & + & - & + & + & L.plantarum \\
\hline 16 & G3 & + & - & - & - & - & - & + & - & + & + & + & - & + & + & - & + & + & L.plantarum \\
\hline 17 & G5 & + & - & - & - & - & - & + & - & + & + & + & - & + & + & - & + & + & L.plantarum \\
\hline 18 & G6 & + & - & - & - & - & - & + & d & + & + & + & - & + & + & - & + & d & $\begin{array}{l}\text { Other Lactobacillus } \\
\text { spp }\end{array}$ \\
\hline 19 & S1 & + & - & - & - & - & - & - & + & - & - & + & d & - & + & d & + & - & Pediococcus Spp \\
\hline 20 & S3 & + & - & - & - & - & - & + & d & + & + & + & - & + & + & - & + & d & $\begin{array}{l}\text { Other Lactobacillus } \\
\text { spp }\end{array}$ \\
\hline 21 & S9 & + & - & - & - & - & - & + & - & + & + & + & - & + & + & - & + & + & L.plantarum \\
\hline 22 & S10 & + & - & - & - & - & - & + & - & + & + & + & - & + & + & - & + & + & L.plantarum \\
\hline
\end{tabular}

Note: d- Doubtful 
Table.2 Detection of LAB isolated from raw milk samples by MALDI-TOF MS

\begin{tabular}{|c|c|c|c|c|}
\hline $\begin{array}{l}\text { Sr } \\
\text { No. }\end{array}$ & Score Value & Detected species & $\begin{array}{l}\text { No. of } \\
\text { species }\end{array}$ & Percentage \\
\hline 1 & $2.301-2.129$ & L. plantarum & 10 & 45.45 \\
\hline 2 & $2.195-2.032$ & Pediococcus acidilactici & 05 & 22.72 \\
\hline 3 & $2.227-2.024$ & $\begin{array}{l}\text { L. plantarum ssp } \\
\text { plantarum }\end{array}$ & 02 & 09.09 \\
\hline 4 & $2.123-1.848$ & L. paraplantarum & 02 & 09.09 \\
\hline 5 & 2.207-1.91 & $\begin{array}{l}\text { L. plantarum ssp } \\
\text { argentoratensis }\end{array}$ & 02 & 09.09 \\
\hline 6 & $0.00-1.142$ & Unidentified & 01 & 04.54 \\
\hline \multicolumn{3}{|r|}{ Total } & 22 & 100 \\
\hline
\end{tabular}

Table.3 Selective potent Lactobacillus isolates having highest activity in Agar spot test

\begin{tabular}{|c|c|c|c|c|c|}
\hline $\begin{array}{l}\text { Sr } \\
\text { no. }\end{array}$ & Source & $\begin{array}{l}\text { Total no of } \\
\text { positive } \\
\text { isolates }\end{array}$ & $\begin{array}{c}\text { Isolate } \\
\text { with } \\
\text { potency }\end{array}$ & Potent Isolate (code) & $\begin{array}{c}\text { Zone of } \\
\text { inhibition } \\
(\mathrm{mm})\end{array}$ \\
\hline 1 & Cow & 04 & 01 & L. paraplantarum $(\mathrm{C} 2)$ & 16 \\
\hline \multirow{5}{*}{2} & \multirow{5}{*}{ Buffalo } & \multirow{5}{*}{09} & \multirow{5}{*}{05} & $\begin{array}{l}\text { L. plantarum ssp } \\
\text { argentoratensis (B1) }\end{array}$ & $13-14$ \\
\hline & & & & L. plantarum (B5) & 15 \\
\hline & & & & $\begin{array}{l}\text { L. plantarum ssp } \\
\text { argentoratensis (B6) }\end{array}$ & 14 \\
\hline & & & & L. plantarum (B8) & 18 \\
\hline & & & & L. paraplantarum (B9) & 17 \\
\hline \multirow{3}{*}{3} & \multirow{3}{*}{ Goat } & \multirow{3}{*}{05} & \multirow{3}{*}{03} & L.plantarum $(\mathrm{G} 1)$ & $14-15$ \\
\hline & & & & L.plantarum (G2) & 15 \\
\hline & & & & L. plantarum (G6) & 18 \\
\hline \multirow{2}{*}{4} & \multirow{2}{*}{ Sheep } & \multirow{2}{*}{04} & \multirow{2}{*}{02} & L. plantarum (S3) & 09 \\
\hline & & & & L. plantarum (S10) & 11 \\
\hline & TOTAL & 22 & 11 & & \\
\hline
\end{tabular}


Table.4 Zones of Inhibition of individual CFS of LAB against COPS MRSA by well diffusion method

\begin{tabular}{|c|c|c|c|c|c|c|c|c|c|c|c|}
\hline \multirow{2}{*}{$\begin{array}{l}\text { CFS } \\
\text { Lactob } \\
\text { acillus } \\
\text { spp. }\end{array}$} & \multicolumn{11}{|c|}{ Mean zone of Inhibition (mm diameter) } \\
\hline & 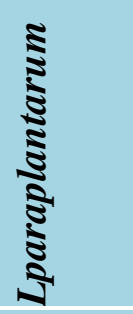 & 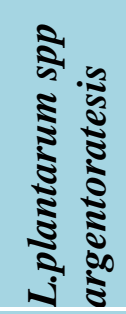 & $\frac{\sqrt{3}}{\sqrt[3]{5}}$ & 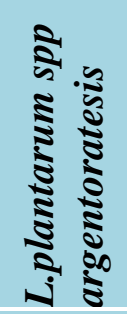 & $\begin{array}{c}5 \\
\frac{5}{5} \\
\frac{5}{5} \\
5 \\
5 \\
5\end{array}$ & 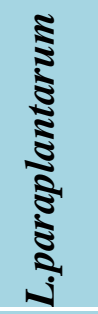 & $\frac{\sqrt{3}}{0}$ & $\frac{\sqrt{3}}{\frac{3}{3}}$ & $\frac{\sqrt{5}}{\sqrt[5]{5}}$ & $\frac{\sqrt{3}}{\sqrt[3]{5}}$ & $\frac{\sqrt{3}}{\frac{5}{5}}$ \\
\hline S1 & 04 & 02 & 10 & 07 & 10 & 02 & 04 & 18 & 13 & 02 & 12 \\
\hline S3 & 09 & 07 & 02 & 11 & 17 & 04 & 02 & 11 & 07 & 06 & 10 \\
\hline S4 & 02 & 09 & 14 & 09 & 14 & 08 & 06 & 15 & 18 & 09 & 04 \\
\hline S5 & 10 & 11 & 11 & 10 & 02 & 04 & 02 & 08 & 10 & 02 & 04 \\
\hline S6 & 08 & 08 & 10 & 04 & 19 & 09 & 08 & 18 & 13 & 09 & 02 \\
\hline S9 & 02 & 05 & 08 & 07 & 11 & 02 & 04 & 12 & 02 & 13 & 07 \\
\hline $\mathrm{S} 10$ & 07 & 02 & 14 & 05 & 08 & 04 & 07 & 02 & 10 & 11 & 06 \\
\hline S11 & 10 & 04 & 11 & 02 & 15 & 07 & 09 & 12 & 11 & 06 & 04 \\
\hline S13 & 05 & 09 & 05 & 09 & 04 & 02 & 02 & 17 & 10 & 11 & 14 \\
\hline S14 & 09 & 07 & 10 & 11 & 12 & 02 & 10 & 09 & 17 & 02 & 06 \\
\hline $\mathrm{S} 16$ & 02 & 06 & 16 & 04 & 12 & 07 & 11 & 18 & 06 & 04 & 04 \\
\hline S17 & 10 & 12 & 11 & 02 & 17 & 04 & 02 & 12 & 14 & 07 & 06 \\
\hline S18 & 04 & 02 & 02 & 08 & 15 & 02 & 06 & 09 & 12 & 11 & 04 \\
\hline S21 & 02 & 12 & 18 & 07 & 10 & 02 & 14 & 17 & 06 & 08 & 11 \\
\hline $\mathbf{S 2 3}$ & 12 & 02 & 11 & 09 & 16 & 04 & 02 & 04 & 15 & 09 & 12 \\
\hline $\mathbf{S 2 6}$ & 07 & 10 & 16 & 11 & 02 & 02 & 07 & 15 & 06 & 07 & 08 \\
\hline $\mathbf{S} 27$ & 09 & 07 & 10 & 02 & 17 & 07 & 05 & 12 & 10 & 04 & 06 \\
\hline S28 & 02 & 05 & 14 & 05 & 11 & 06 & 10 & 08 & 02 & 08 & 07 \\
\hline S29 & 02 & 04 & 15 & 10 & 17 & 12 & 04 & 15 & 14 & 11 & 02 \\
\hline S30 & 05 & 02 & 09 & 05 & 02 & 04 & 12 & 02 & 17 & 11 & 04 \\
\hline S33 & 07 & 10 & 12 & 02 & 16 & 08 & 02 & 11 & 04 & 02 & 11 \\
\hline S35 & 02 & 11 & 10 & 05 & 14 & 02 & 09 & 08 & 12 & 09 & 10 \\
\hline $\begin{array}{c}\text { Mean } \pm \\
\text { SE }\end{array}$ & $\begin{array}{c}5.91 \\
\pm 0.72^{b} \\
c\end{array}$ & $\begin{array}{l}6.68 \pm \\
0.74^{\text {bc }}\end{array}$ & $\begin{array}{r}10.86 \\
\pm 0.88^{a}\end{array}$ & $\begin{array}{l}6.59 \pm \\
0.67^{\mathrm{bc}}\end{array}$ & $\begin{array}{c}11.6 \\
\pm 1.13^{a}\end{array}$ & $\begin{array}{l}4.73 \\
\pm 0.6 \\
1^{c}\end{array}$ & $\begin{array}{c}6.27 \\
\pm 0.79^{b c}\end{array}$ & $\begin{array}{c}11.5 \\
\pm 1.05^{\mathrm{a}}\end{array}$ & $\begin{array}{c}10.4 \\
\pm 1.0 \\
a\end{array}$ & $\begin{array}{c}7.363 \\
\pm 0.73 \\
b\end{array}$ & $\begin{array}{c}7 \pm 0 \\
747^{b} \\
\text { c }\end{array}$ \\
\hline $\begin{array}{l}\text { Level } \\
\text { of } \\
\text { signifi } \\
\text { cance }\end{array}$ & $* *$ & $* *$ & $*$ & $* *$ & $*$ & $* *$ & $* *$ & $*$ & $*$ & $* *$ & $* *$ \\
\hline
\end{tabular}

Note: a, b, c Means with different superscripts in a column differ significantly $* \mathrm{P}<0.05 ; * * \mathrm{P}<0.01$ 
Table.5 Mean Inhibition zone of lactobacilli against $S$. aureus by agar disc diffusion method

\begin{tabular}{|c|c|c|c|c|c|c|c|c|c|c|c|}
\hline $\begin{array}{l}\text { CSF of } \\
\text { Lactobacillu } \\
s \text { species }\end{array}$ & \multicolumn{11}{|c|}{ Inhibition zone diameter ( $\mathrm{mm}$ ) } \\
\hline $\begin{array}{l}s \text { species } \\
\text { MRSA }\end{array}$ & 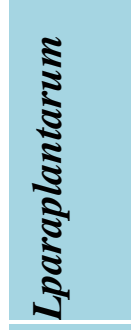 & 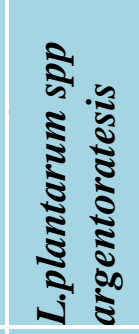 & $\frac{\sqrt[3]{3}}{3}$ & 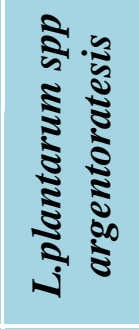 & $\frac{\sqrt{3}}{3}$ & 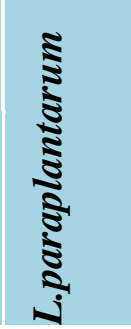 & $\frac{\sqrt{3}}{3}$ & 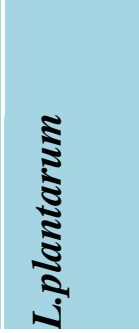 & 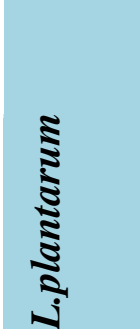 & 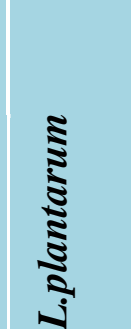 & 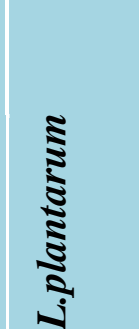 \\
\hline S1 & 12 & 4 & 11 & 14 & 14 & 02 & 02 & 02 & 11 & 02 & 10 \\
\hline S3 & 07 & 09 & 04 & 09 & 17 & 02 & 04 & 07 & 06 & 16 & 11 \\
\hline S4 & 12 & 07 & 11 & 08 & 12 & 07 & 04 & 02 & 08 & 09 & 02 \\
\hline S5 & 09 & 07 & 15 & 05 & 04 & 02 & 02 & 08 & 09 & 12 & 02 \\
\hline S6 & 07 & 5 & 19 & 12 & 17 & 09 & 07 & 06 & 11 & 09 & 02 \\
\hline S9 & 11 & 07 & 07 & 05 & 19 & 02 & 04 & 09 & 02 & 11 & 07 \\
\hline$\overline{\text { S10 }}$ & 07 & 02 & 12 & 15 & 07 & 02 & 05 & 02 & 09 & 09 & 07 \\
\hline S11 & 07 & 02 & 19 & 12 & 12 & 05 & 07 & 11 & 09 & 17 & 02 \\
\hline$\overline{\text { S13 }}$ & 15 & 07 & 13 & 09 & 12 & 02 & 04 & 07 & 09 & 09 & 11 \\
\hline S14 & 16 & 05 & 17 & 09 & 11 & 02 & 07 & 07 & 05 & 12 & 05 \\
\hline S16 & 02 & 05 & 09 & 14 & 14 & 05 & 09 & 07 & 07 & 02 & 02 \\
\hline S17 & 16 & 09 & 11 & 12 & 02 & 05 & 02 & 04 & 11 & 17 & 05 \\
\hline S18 & 02 & 04 & 12 & 08 & 15 & 02 & 05 & 07 & 11 & 09 & 02 \\
\hline S21 & 12 & 09 & 15 & 12 & 19 & 04 & 11 & 07 & 03 & 17 & 07 \\
\hline S23 & 09 & 02 & 09 & 07 & 15 & 02 & 02 & 04 & 04 & 17 & 07 \\
\hline S26 & 15 & 09 & 12 & 09 & 02 & 02 & 05 & 02 & 07 & 07 & 05 \\
\hline$\overline{S 27}$ & 12 & 05 & 18 & 12 & 12 & 07 & 05 & 02 & 09 & 12 & 05 \\
\hline S28 & 02 & 03 & 15 & 12 & 19 & 05 & 09 & 07 & 02 & 12 & 07 \\
\hline S29 & 03 & 02 & 14 & 07 & 17 & 11 & 04 & 02 & 02 & 11 & 02 \\
\hline S30 & 12 & 02 & 07 & 12 & 12 & 02 & 11 & 02 & 05 & 09 & 02 \\
\hline S33 & 15 & 09 & 12 & 02 & 15 & 09 & 02 & 09 & 02 & 12 & 09 \\
\hline S35 & 02 & 02 & 02 & 02 & 17 & 02 & 07 & 07 & 11 & 09 & 09 \\
\hline Mean \pm SE & $\begin{array}{l}9.32 \\
\pm 1.04^{b}\end{array}$ & $\begin{array}{l}5.27 \pm \\
0.57^{b c}\end{array}$ & $\begin{array}{l}12 \pm \\
0.96^{\mathrm{a}}\end{array}$ & $\begin{array}{r}9.40 \\
\pm 0.78^{b}\end{array}$ & $\begin{array}{l}12 \pm \\
0.96^{\mathrm{a}}\end{array}$ & $\begin{array}{l}4.13 \\
\pm 0.60^{c}\end{array}$ & $\begin{array}{l}5.36 \\
\pm 0.60^{c}\end{array}$ & $\begin{array}{l}5.5 \\
\pm 0.61^{\text {bc }}\end{array}$ & $\begin{array}{l}6.95 \pm 0 . \\
71^{\mathrm{bc}}\end{array}$ & $\begin{array}{l}10.9 \\
\pm 0.91^{a}\end{array}$ & $\begin{array}{l}5.5 \\
\pm 0.68^{b c}\end{array}$ \\
\hline $\begin{array}{l}\text { Level of } \\
\text { significance }\end{array}$ & $* *$ & $* *$ & $*$ & $* *$ & $*$ & $* *$ & $*$ & $* *$ & $* *$ & $*$ & $* *$ \\
\hline
\end{tabular}

Note: a, b, c Means with different superscripts in a column differ significantly $* \mathrm{P}<0.05$; 
Plate.1 Zone of inhibition of Lactobacillus spp. in Agar spot assay

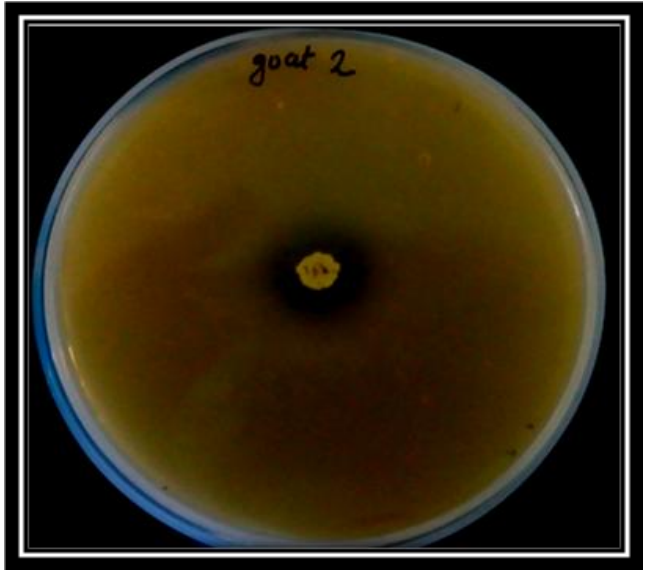

Plate.2 Zones of inhibition of LAB in Agar well diffusion test

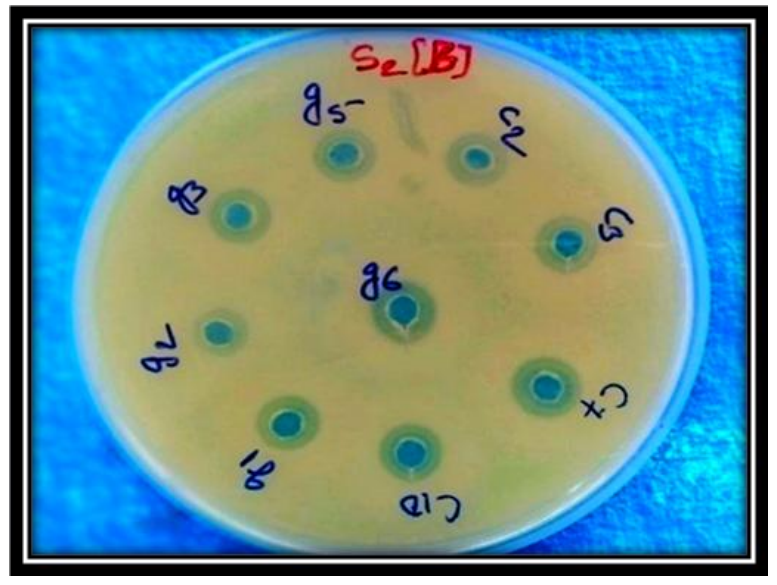

Plate.3 Zones of inhibition of LAB in Agar well diffusion test

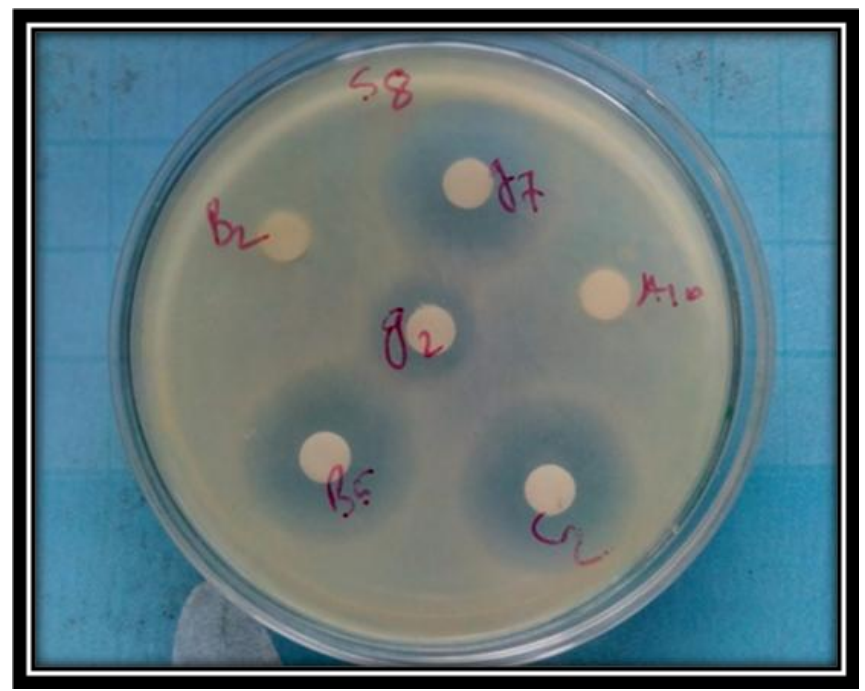


In sugar fermentation test 12 out of $22 \mathrm{LAB}$ fermented Cellobiose, Mannitol, Melebiose, Raffinose, Ribose, Salicine, Trehalose and Sucrose, but did not ferment Arabinose, Lactose and Sorbitol, therefore were identified as L. plantarum. Six isolates fermented Cellobiose, Mannitol, Melebiose, Raffinose, Ribose, Salicine, but not Lactose and Sorbitol and results of Arabinose and Trehalose remained doubtful, hence, were grouped as other non typable Lactobacillus spp. Remaining four isolates fermented Melabiose, Raffinose, Ribose, Arabinose, Trehalose but not Cellobiose and Mannitol, and results of Lactose and Sorbitol were doubtful hence, they were grouped as Pediococcus spp. Our findings were consistency with the Mohankumar and Murugalatha (2011) and Sarangdhar et al., (2015).

\section{Molecular identification of LAB by MALDI-TOF MS}

Employing the MALDI-TOF MS technique LAB species were identified as per table 2; out of which highest number of isolates 10/22 were identified as L. plantarum $(72.72 \%)$ while other six were subspecies of $L$. plantarum identified as two L. plantarum ssp plantarum $(00.09 \%)$, two L. plantarum ssp argentoratensis (09.00\%) and two $L$. paraplantarum $(09.00 \%)$ strains.

Another 05/22 (22.72\%) isolates identified as Pediococcus spp. in cultural method were confirmed with MALDI TOF MS technique as Pediococcus acidilactici. While one isolate out of 22 remained unidentified as peak score observed was not optimum. The results of ten bacterial isolate identified as $L$. plantarum with cultural method and genomic PCR remained in cent per cent agreement with MALDI-TOF MS which was in close accordance with Dogan and Ozpinar (2017) and Dissanayake (2017).

\section{Antimicrobial activity}

\section{Agar spot assay}

As per the results in the table 3 of Agar Spot assay, out of four positive isolates of LAB sourced from cow only one $(18.18 \%)$ i.e. $L$. paraplantarum (C2) was found highly effective, five out of nine isolates belonging to buffaloes $(36.36 \%)$ i.e. two L. plantarum (B5 and B8), one L. paraplantarum (B9) and two L. plantarum spp argentoratensis (B1 and B6), three out of five isolates belonging goat (27.27\%) i.e. L. plantarum (G1, G2 and G6) and two out of four isolates belonging to sheep raw milk $(13.60 \%)$ i.e. L. plantarum (S3 and S10) were having the highest activity. Hence, these isolates were selected for further assessment of their in vitro antibacterial efficacy against COPS MRSA. Our findings closely corroborated with earlier researchers Mohankumar and Murugalatha (2011) and Jothi et al., (2012).

\section{Antibacterial efficacy of LAB against COPS MRSA with Agar Well Diffusion} Test

The findings of antibacterial activity of CFS of LAB against COPS MRSA by well diffusion method were as depicted in table 3 (Plate 2). The widest zone of inhibitions (15$18 \mathrm{~mm})$ was recorded with strains $L$. plantarum (B5 and B8) isolated from buffalo followed by L. plantarum (G2 and G6) from goats $(10-14 \mathrm{~mm})$. This trend was also seen in sheep L. plantarum (S3). Moderate size zones (06-09 mm) were recorded with $L$. paraplantarum (C2) isolated from cow. Overall, out of eleven L. plantarum, four strains (B5, B8, G2 and G6) presented very strong to strong antibacterial efficacy and three strains; L. paraplantarum, L. plantarum spp argentoratesis and L. plantarum showed moderate zone of inhibition ( $\mathrm{C} 2, \mathrm{~B} 6$ and S3). Remaining Lactobacillus species recorded 
small zones of inhibition of around $05 \mathrm{~mm}$. Thus present findings remained in close concurrence with Soleimani et al., (2010) and Eid et al., (2016).

Statistically, the results of treatments with Lactobacillus species against COPS MRSA were found significantly different at $1 \%$ level of significance at the P-value less than 0.01 .

\section{Antibacterial efficacy of LAB against COPS MRSA with Agar Disc Diffusion Test}

The antibacterial activity CFS of LAB against COPS MRSA was also examined by disc diffusion method to support the results of agar well diffusion test and the results were as depicted in table 4 (Plate. 3) which indicated that L. plantarum (B5, B8, G2 and G6) obtained from buffaloes and goats exhibited highest anti MRSA activity (15-18 $\mathrm{mm}$ zone of inhibition), followed by moderate efficacy (10-12 $\mathrm{mm}$ zone of inhibition) by $L$. plantarum (S3) and L. paraplantarum (C2) from sheep and cow, respectively (Table 5).

Our results were in accordance with the earlier report on antagonistic effect of LAB on gram positive and gram negative bacteria of Tajehmiri and co-workers (2014).

The statistical analyses of results of the treatments were found significant at $1 \%$ level of significance when the P-value were less than 0.01 .

L. plantarum isolated from buffaloe and goats and L. paraplantarum from cows recorded the strongest antimicrobial efficacy against the virulent MRSA, and even L. plantarum from sheep also exhibited some efficacy, hence, the Lactobacillus strains in our study were found to be potential candidates for further exploiting their antibacterial efficacy against virulent MRSA in vivo.

\section{Acknowledgment}

Authors are grateful to the Department of Veterinary Microbiology, Shirwal, MAFSU, Nagpur (MS), India for providing all the necessary support and facility.

\section{References}

Asfour, H. A. E. and S. F. Darwish (2011) Phenotypic and genotypic detection of both $M e c A$ and blaZ genes mediated $\beta$ lactam resistance in Staphylococcus strains isolated from bovine mastitis. Global Veterinaria. 6 (1): 39-50.

Bauer, A.W., W. M. M. Kirby, J. C. Sherris and M. Turck (1966) Antibiotic susceptibility testing by a standardized single disc method. American J. Clin. Pathol. 45: 493-496.

Behiry, A. E., G. Schlenker, I. Szabo, U. Roesler (2012) In vitro susceptibility of Staphylococcus aureus strains isolated from cows with subclinical mastitis to different antimicrobial agents. J. Vet. Sci.13 (2):153-161.

Bergey's manual of systemic bacteriology, 1986.

Chandrasekaran, D., P. Venkatesan, K. G. Tirumurugaan, A. P. Nambi, P. S. Thirunavukkarasu, K. Kumanan, S. Vairamuthu and S. Ramesh (2014a) Pattern of antibiotic resistant mastitis in dairy cows. Vet. World 7(6): 389-394.

Chandrasekaran, D., P. Venkatesan, K. G. Tirumurugaan, A. P. Nambi, P. S. Thirunavukkarasu, K. Kumanan and S. Vairamuthu (2014b) A study on Methicillin resistant Staphylococcus aureus mastitis in dairy cows. J. of Appl. Natural Sci.6 (2): 356-361.

CLSI (2014) Performance standards for antimicrobial susceptibility testing -24 the informational supplement M100S24 Clinical Laboratory Standard Institute. M100-s27., 282. 
Coeuret, V., S. Dubernet, M. Bernardeau, M. Gueguen and J. P. Vernoux (2003) Isolation, characterization and identification of lactobacilli focusing mainly on cheeses and other dairy product. EDP Scie. Lait (83) 269-306.

Eid, R., E. J. Jakee, A. Pashidy, H. Asfour, S. Omara, M. M. Kandil, Z. Mahmood, J. Hahne and A. A. Seida (2016) Potential Antimicrobial Activity Of Probiotic Lactobacillus Strain Isolated from Raw Milk in Egypt 4: 2.

Esslemont, R. J., M. A. Kossaibati and J. Allcock (2000) Economics of fertility in dairy cows. Occasional publication. Animal Sci., 26: 19-29.

Gentilini, E., G. Denamiel, P. Llorente, S. Godaly, M. Rebuelto, and O. DeGregorio (2000) Antimicrobial Susceptibility of Staphylococcus aureus isolated from bovine mastitis in Argentina. J. Dairy Sci. 83: 1224-1227.

Hamed, M. I. and A. M. A. Zaitoun (2014) Prevalence of Staphylococcus aureus Subclinical mastitis in Dairy Buffaloes Farms, International Journal of Livestock Research, 4 (3): 22- 28.

Mohankumar and Murugalatha (2011) Characterization and Antibacterial Activity of Bacteriocin Producing Lactobacillus Isolated From Raw Cattle Milk Sample in Tamil Nadu, India International J. of Biology 3(3): 614028

Nowroozi, J., M. Mirzaii and M. Norouzi (2004) Study of Lactobacillus as probiotic bacteria. Iran J. Public Health 33(2): 1-7.

Saidi, R., D. Khelef, and R. Kaidi (2013) Subclinical mastitis in cattle in Algeria: frequency of occurrence and bacteriological isolates. J. South African Vet. Asso. 84 (1): 1-5.

Sarangdhar Mithun, D. Vora and S. Sarang (2015) Screening and Characterization Of Novel Bacteriocin Producing Lactobacilli Obtained From Raw Milk Samples, World Journal of Pharmaceutical Research Vol. 4 (4): 2277- 7105 .

Sharma, L., A. K. Verma, A. Kumar, A. Rahat, Neha and R. Nigam (2015) Incidence and Pattern of Antibiotic Resistance of Staphylococcus aureus Isolated from Clinical and Subclinical Mastitis in Cattle and Buffaloes. Asian J. of Anim. Sci. 9(3): 100-109.

Soleimani, N., K. Rooha, B. Yakhchali and T. Nejad (2010) Antagonistic activity of probiotic lactobacilli against staphylococcus aureus Isolated from bovine Mastitis, Iran.4 (20), PP. 21692175.

Tajehmiri, A., R. K. Darsanaki, M. N. Moslem, Z. Lozoumi, M. H. Kolavani and M. A. Aliabadi (2014) Antimicrobial activity of Lactobacillus spp. Isolated from commercial yoghurts against pathogenic bacteria. J. of Pure and Appl. Microbiol, vol. 8(3), pp. 2211-221.

\section{How to cite this article:}

Jyotika D. Sangle, Prashant P. Mhase, Mrunalini M. Pawade and Prerana R. Shelke. 2018. Antimicrobial Activity of Lactobacilli against Staphylococcus aureus MRSA Strains Isolated from Mastitis in Dairy Animals. Int.J.Curr.Microbiol.App.Sci. 7(11): 3201-3213. doi: https://doi.org/10.20546/ijcmas.2018.711.368 